\title{
3D-confocal structural analysis of bone marrow-derived renal tubular cells during renal ischemia/reperfusion injury
}

\author{
Hideyoshi Toyokawa ${ }^{1,2}$, Atsunori Nakao, ${ }^{1,2}$, Donna B Stolz ${ }^{3}$, Anna J Romanosky ${ }^{3}$, \\ Michael A Nalesnik ${ }^{1,4}$, Joao Seda Neto ${ }^{1,2}$, Takashi Kaizu ${ }^{1,2}$, Anthony J Demetris ${ }^{1,4}$ \\ and Noriko Murase ${ }^{1,2}$
}

${ }^{1}$ Thomas E Starzl Transplantation Institute, University of Pittsburgh Medical Center, Pittsburgh, PA, USA; ${ }^{2}$ Department of Surgery, University of Pittsburgh Medical Center, Pittsburgh, PA, USA; ${ }^{3}$ Center for Biologic Imaging, University of Pittsburgh Medical Center, Pittsburgh, PA, USA and ${ }^{4}$ Department of Pathology, University of Pittsburgh Medical Center, Pittsburgh, PA, USA

\begin{abstract}
Bone marrow cells (BMC) have been shown to migrate into injured sites for parenchymal repair. However, the extent of BMC involvement is controversial. To determine whether and to what extent BMC contribute to renal parenchymal repair, we employed three-dimensional (3D) fluorescent confocal microscopy/video in renal warm and cold ischemia/reperfusion (I/R) injury using enhanced green fluorescent protein transgenic rats and their radiation chimeras. After induction of renal warm I/R injury in chimeras, BM-derived renal tubular cells were found in 2D microscopy as isolated single cells or clusters of 2-3 cells. Likewise, cold I/R injury resulted in hostderived tubular cells with frequencies $\sim 0.2 \%$. However, stringent confocal microscopic analysis and 3D image construction revealed that BM-derived tubules identified in 2D images were frequently artifacts of overlapping cells separately stained with different markers. The actual frequency in 3D analysis was approximately onefourth of that seen in 2D analysis. 3D confocal imaging precisely detected BM-derived tubular epithelial cells and could be useful to study BMC contribution to tissue repair.
\end{abstract}

Laboratory Investigation (2006) 86, 72-82. doi:10.1038/labinvest.3700363; published online 24 October 2005

Keywords: ischemia/reperfusion injury; tissue regeneration; bone marrow-derived cells; three dimension; kidney transplantation; GFP

Recent studies have suggested that adult bone marrow cells (BMC) have far more plasticity than previously implied. It had been thought that stem cells can only differentiate into certain restricted lineages of mature cells. However, newer studies have demonstrated an expanding repertoire of BMC; they are capable of crossing lineage barriers and adopting the phenotypic profiles and functional properties of cells unique to other tissues. BMC can differentiate into various lineages, including epithelium of the liver, lung, and gut, myocytes, neural cells, and renal tubular cells..$^{1-5}$

Although there are abundant data on the phenotype, isolation, culturing, expansion, and differentiation potential of stem cells in the bone marrow, their role and functional relevance in normal adult

Correspondence: Dr N Murase, MD, Thomas E Starzl Transplantation Institute, Department of Surgery, E1555 Biomedical Science Tower, University of Pittsburgh, Pittsburgh, PA 15213, USA.

E-mail: murase@pitt.edu

Received 9 June 2005; revised 13 September 2005; accepted 20 September 2005; published online 24 October 2005 life or regenerative parenchymal cell turnover is unclear. In addition, many of the findings in this new field are controversial, partly due to the inherent difficulties of techniques used to assess the plasticity of these cells.

In this study, transgenic rats carrying the enhanced green fluorescent protein (EGFP) transgene were used to demonstrate three-dimensional (3D) structure analysis of the differentiation of BMC to renal tubular epithelial cells after renal ischemia/ reperfusion (I/R) injury. The results suggest that BMderived cells can differentiate into renal tubular epithelial cells after I/R injury; however, the frequency is considerably less with $3 \mathrm{D}$ analysis than that identified with conventional 2D microscopic analyses of thin sections.

\section{Materials and methods}

Animals

EGFP-transgenic and WT Sprague-Dawley (SD) rats, originally generated by Dr Masaru Okabe (University 
of Osaka, Osaka, Japan), ${ }^{6,7}$ were obtained from Japan SLC, Inc. (Hamamatsu, Japan). The expression of EGFP was under the control of the cytomegalovirus enhancer and the chicken $\beta$-actin promoter derived from an expression vector, pCAGGS. ${ }^{6,7}$ Animals were maintained in laminar flow cages in a specific pathogen-free animal facility at the University of Pittsburgh. All procedures in this experiment were performed according to the guidelines of the Council on Animal Care at the University of Pittsburgh and the National Research Council's Guide for the Humane Care and Use of Laboratory Animals.

\section{Creation of Radiation Chimeras and Hematopoietic Reconstitution}

BMC were obtained from 8-12-week-old EGFP or WT rats by flushing the tibias and femurs with RPMI 1640, supplemented with $25 \mathrm{mM}$ HEPES buffer, $2 \mathrm{mM}$ L-glutamine, and $10 \mu \mathrm{g} / \mathrm{ml}$ gentamicin (all from Life Technologies, Grand Island, NY, USA). Unfractionated BMC $\left(2 \times 10^{8}\right.$ cells/animal $)$ with $>95 \%$ viability in trypan blue exclusion test were intravenously injected into EGFP or WT rat recipients after $9.5 \mathrm{~Gy}$ whole body irradiation (WBI). ${ }^{8,9}$ Tacrolimus $(0.5 \mathrm{mg} / \mathrm{kg} /$ day, a gift from Fujisawa Pharmaceutical Co., Osaka, Japan) was given for 7 days after BMC infusion. Development of hematolymphoid cells from infused BMC in radiation chimeras was detected by flow cytometry with mouse monoclonal antibody $(\mathrm{mAb})$ phycoerythrin (PE)-Cy5-conjugated OX-1 (CD45, Pharmingen, San Diego, CA, USA) and tissue sections with immunofluorescent analysis. In addition, polyclonal rabbit anti-GFP antibody (Molecular Probes, Inc., Eugene, OR, USA) was used to detect EGFP-expressing cells in the immunoperoxidase method.

\section{Induction of Renal I/R Injury}

\section{Warm I/R injury in radiation chimeras}

At $>100$ days after BMC infusion, radiation chimeras with $>95 \%$ donor hematolymphoid cells underwent warm renal I/R injury. As described previously, ${ }^{10}$ the right renal artery was isolated and clamped for $60 \mathrm{~min}$. The left kidney was removed at the time of warm I/R injury or 7 days later.

\section{Cold I/R injury with orthotopic kidney \\ transplantation (KTX)}

Orthotopic KTx was performed, as described previously. ${ }^{11}$ The donor left kidney was flushed with $3 \mathrm{ml}$ University of Wisconsin (UW) solution (Du Pont, Wilmington, DE, USA) and transplanted either immediately or after preservation in UW at $4{ }^{\circ} \mathrm{C}$ for $18 \mathrm{~h}$. The kidney graft was transplanted into the recipient by end-to-side anastomoses between the graft aorta and recipient infrarenal abdominal aorta, and between the graft renal vein and recipient infrarenal vena cava. The recipient left native kidney was removed, and end-to-end ureteral anastomosis was performed. The right native kidney was removed at the time of KTx for short-term study or 7 days after KTx for long-term study.

\section{Samples after Renal I/R Injury}

At 1,7 , and 30 days after warm or cold renal I/R injury, blood samples were obtained for serum creatinine levels, and kidney samples were obtained by perfusing animals via the abdominal aorta with phosphate-buffered saline (PBS), followed with $2 \%$ paraformaldehyde in PBS. EGFP protein is designed to be expressed in the cytosol and the soluble nature of the protein allows its distribution through the cytosol. ${ }^{6,7}$ Therefore, snap-freezing of samples without fixation resulted in a significant reduction of the green signal due to leaching of EGFP protein out of the cells during the thaw process. To avoid this problem, all tissues were perfusion fixed with $2 \%$ paraformaldehyde. The kidney samples were removed and stored in $2 \%$ paraformaldehyde for several hours at $4^{\circ} \mathrm{C}$ for the histopathological assays described below.

\section{Histopathological Analyses}

Routine histopathology

Tissue samples were embedded in paraffin wax, sectioned at $5 \mu \mathrm{m}$, and stained with hematoxylin and eosin.

\section{Immunofluorescence and image analysis}

Tissue samples were cryoprotected in $2.3 \mathrm{M}$ sucrose in PBS overnight, embedded in OCT compound, and frozen in liquid nitrogen-cooled isopentane. Samples were cut into 6 - or $50-\mu \mathrm{m}$ sections on gelatincoated slides, washed with PBS containing $0.5 \%$ bovine serum albumin and $0.15 \%$ glycine (PBG buffer), and fixed with $2 \%$ paraformaldehyde. After blocking with $20 \%(\mathrm{v} / \mathrm{v})$ normal goat serum in PBG, 6- and 50- $\mu \mathrm{m}$ sections were stained with rhodamine phalloidin (1:1000, Molecular Probes, Inc., Eugene, OR, USA), which bound with a nanomolar affinity to F-actin, for 30 and $90 \mathrm{~min}$, respectively. Sections of $6 \mu \mathrm{m}$ were nuclear DNA stained with Hoechst dye (bisBenzimide), coverslipped with Gelvatol, visualized with an Olympus BX51 epifluorescence microscope, and 2D images were digitized with an Olympus/Optronics (Goleta, CA, USA) CCD camera, interfaced with MagnaFire Image Capture Software.

Rhodamine phalloidin-stained $50-\mu \mathrm{m}$ sections were nuclear stained with DRAQ5 (Biostatus Limited, Shepshed, Leicestershire, UK), coverslipped with Gelvatol, and viewed with an Olympus FV 500 confocal scanning microscope. Confocal slices were taken at $0.3-\mu \mathrm{m}$ intervals, and a maximum intensity projection and 3D movie was created using Metamorph software (Universal Imaging Corporation ${ }^{\mathrm{TM}}$ ). 
The rhodamine (orange-red) signal was excited at $550 \mathrm{~nm}$ and emission was collected from 590 to $610 \mathrm{~nm}$. EGFP (green) signal, in contrast, was excited at $488 \mathrm{~nm}$ and emission was collected from 510 to $525 \mathrm{~nm}$. Nuclear Hoechst dye and DRAQ5 (blue) signals were excited at $650 \mathrm{~nm}$ and emission was collected from 670 to $700 \mathrm{~nm}$. The fluorescent signals were collected sequentially using the narrow collection wavelengths set up by the confocal microscope software. By using this approach and by setting the photomultiplier tubes correctly, there was no signal bleed through.

\section{Identification and quantification of renal tubules derived from $B M C$}

In cases of EGFP BMC to WT chimeras and WT to EGFP KTx, renal tubular epithelial cells regenerated from $\mathrm{EGFP}^{+} \mathrm{BMC}$ after I/R injury were identified as tubules that contained EGFP ${ }^{+} /$F-actin $^{+} /$Hoechst $^{+}$ cells. Alternatively, in EGFP recipients of WT BMC, tubular epithelial cells regenerated from WT BMC after warm I/R injury were identified as $\mathrm{EGFP}^{-}$/ F-actin ${ }^{+} /$Hoechst $^{+}$cells among $\mathrm{EGFP}^{+} / \mathrm{F}$-actin ${ }^{+} /$ Hoechst $^{+}$cells. The frequency of tubules that contained BM-derived tubular cells was determined by examining 250-1100 randomly selected tubules in the outer medulla of the $6-\mu \mathrm{m}$ sections with $2 \mathrm{D}$ epi-fluorescence microscope analysis. In $50-\mu \mathrm{m}$ sections, $\mathrm{EGFP}^{+} / \mathrm{F}_{\text {-actin }}{ }^{+} / \mathrm{DRAQ}^{+}{ }^{+}$cells were identified initially in the 2D analysis with confocal scanning microscopy, and 3D construction of the suspected target cells was generated by serially slicing at $0.3-\mu \mathrm{m}$ intervals.

\section{Statistical Analyses}

All values in this study are expressed as mean \pm s.d. Student's $t$-test was used for the analyses of the difference between groups. $P$-value less than 0.05 was considered significant.

\section{Results}

\section{Radiation Chimera}

In naïve EGFP rats, $>99 \%$ of $\mathrm{CD}_{4} 5^{+}$peripheral blood mononuclear cells (PBMC) expressed EGFP in flow cytometry (Figure 1a-c). In contrast, PBMC from WT animals were totally negative for EGFP (Figure 1d). After WBI and BMC infusion, the percentage of donor BM-derived PBMC quickly increased and reached $>90 \%$ at 60 days. At 100 days, in WT recipients of $\mathrm{EGFP}^{+} \mathrm{BMC}>95 \%$ of $\mathrm{CD}^{4} 5^{+} \mathrm{PBMC}$ were EGFP ${ }^{+}$(Figure 1e). Likewise, CD45 ${ }^{+}$PBMC in EGFP recipients of WT BMC were negative for EGFP (Figure 1f).

In kidney tissues of radiation chimeras, different origins of nonparenchymal and parenchymal cells were apparent, depending on EGFP expression. In WT recipients of $\mathrm{EGFP}^{+} \mathrm{BMC}$, the kidney sections showed homogenous distribution of $\mathrm{EGFP}^{+}$nonparenchymal cells throughout the renal interstitium, while tubular epithelial cells, glomerular structures, and vasculature were negative for EGFP (Figure 1g). To confirm the presence of $\mathrm{EGFP}^{+}$cells and to eliminate the possibility of autofluorescence, kidney samples were stained with anti-GFP antibody. Results verified the scattered distribution of EGFPexpressing cells in the renal interstitium (Figure 1h). In contrast, in EGFP recipients of WT BMC, kidney sections presented a mirror image; $\mathrm{EGFP}^{-}$leukocytes were found among overwhelming numbers of EGFP-expressing parenchymal cells (tubules, glomeruli, and vessels) (Figure 1i). In both types of radiation chimeras, renal tubular epithelial cells maintained the original recipient phenotype, and we were not able to find BM-derived tubular epithelial cells.

\section{Serum Creatinine Levels after Warm and Cold Renal I/R Injury}

At $24 \mathrm{~h}$ after warm I/R injury, serum creatinine levels quickly elevated to $3.72 \pm 1.11$ from $0.62 \pm$ $0.08 \mathrm{mg} / \mathrm{dl}$ in normal animals. Cold I/R injury with $18 \mathrm{~h}$ preservation resulted in higher creatinine levels of $4.33 \pm 0.31 \mathrm{mg} / \mathrm{dl}$ at $24 \mathrm{~h}$. On the other hand, cold $\mathrm{I} / \mathrm{R}$ injury with minimum preservation showed a marginal creatinine elevation. At 30 days after I/R injury, creatinine levels in warm $I / R$ injury and minimum cold I/R injury returned to normal levels. However, creatinine levels after cold I/R injury in $18 \mathrm{~h}$ preservation model remained higher than normal (Table 1).

\section{Routine Histopathology of Warm and Cold Renal I/R Injury}

The most significant change at 7 days after warm I/R injury was evidence of tubular epithelial cell death manifested by intraluminal necrotic cell debris, scattered apoptosis, and variable tubular dilatation with protein casts. Mild to moderate interstitial inflammation was also present. The changes mainly involved the cortex, with some extension to the outer medulla; however, glomerular injury was not noticeable (Figure 2a). By 30 days, active tubular injury diminished, although variable tubular dilatation and interstitial inflammation persisted (Figure 2d).

After cold I/R injury with a minimum preservation period, tubular epithelial cell vacuolization was noted with minimal to no tubular dilatation and occasional necrotic tubular epithelial cells. Interstitial inflammation ranged from mild to moderate (Figure 2b). These changes were restored and totally repaired by 30 days; kidney grafts showed nearly normal histopathology with some focal inflammation and occasional focal injury attributable to surgery (Figure 2e). 
a

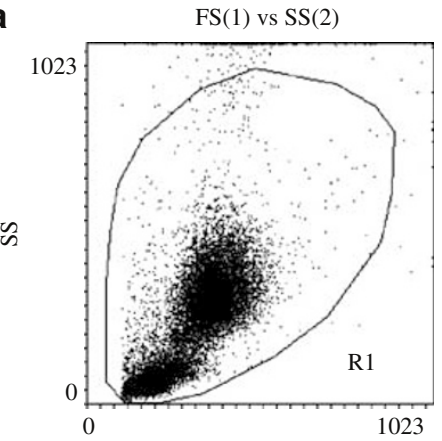

FS

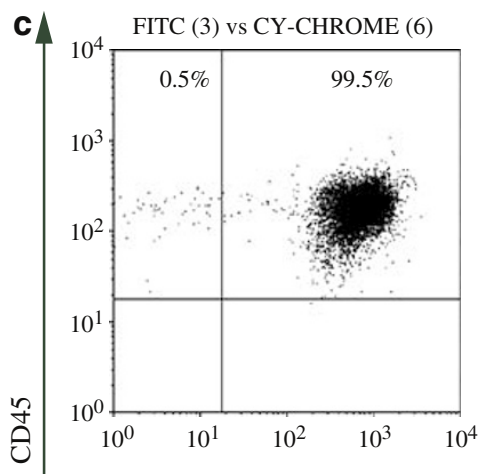

e

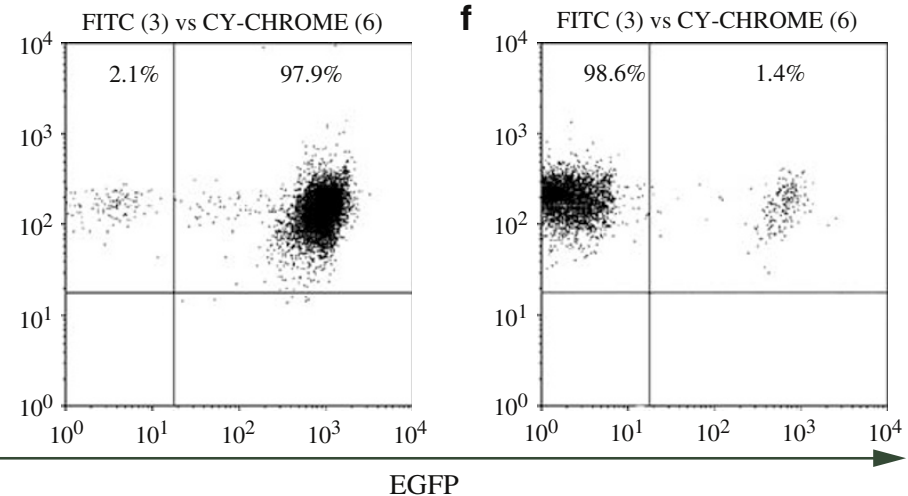

b

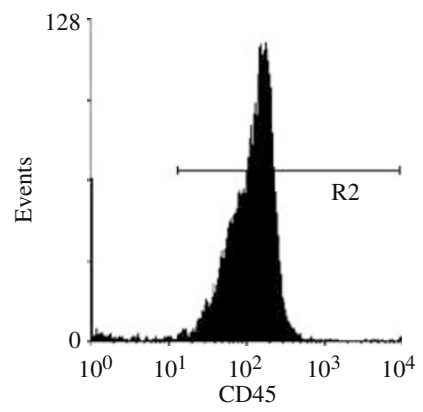

d

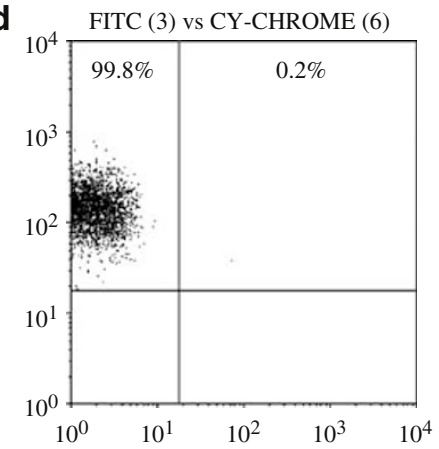

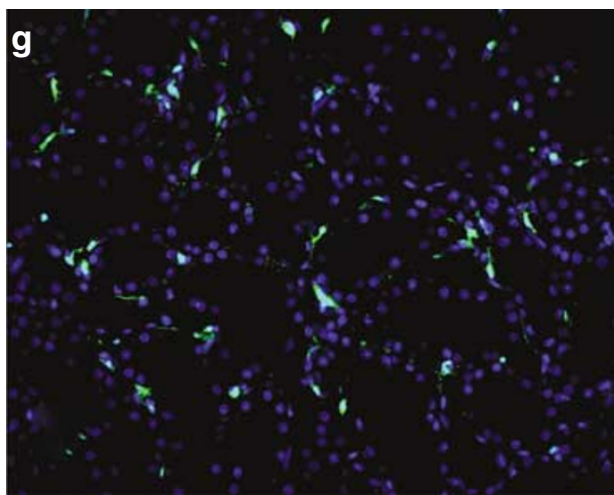
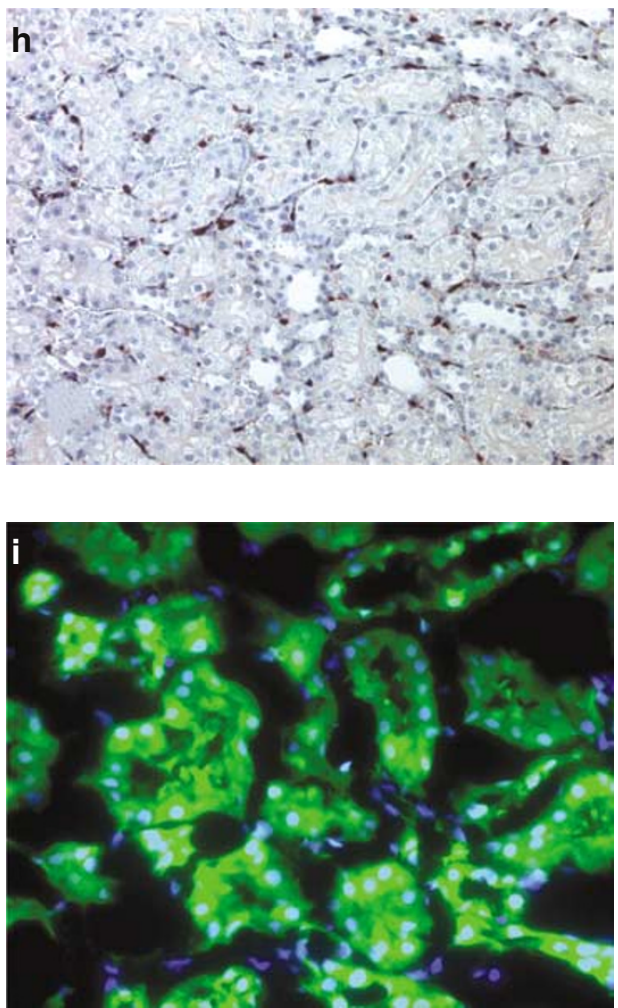

Figure 1 Hematopoietic reconstitution in radiation chimeras. At 100 days after WBI and BMC infusion, phenotypes of PBMC were evaluated by two-color flow cytometry. (a, b) Using side-scatter (SS) vs forward-scatter (FS) cytograms, cells in a whole leukocyte cluster (R1) were positive for mAb OX-1 (CD45). (c) Nearly all (99.5\%) of PBMC in naïve EGFP ${ }^{+}$rats expressed EGFP. (d) PBMC of naïve WT rats were negative for EGFP. (e) WT recipients of EGFP ${ }^{+}$BMC showed 97.9\% EGFP ${ }^{+}$PBMC. (f) Majority (98.6\%) of PBMC from EGFP recipients of WT BMC were negative for EGFP. (g) Frozen kidney sections from WT chimeric rats of EGFP BMC showed scattered EGFP ${ }^{+}$ cells in the interstitial space of outer medulla. Magnification $\times 400$. (h) The same section was stained with the immunoperoxidase method using anti-EGFP mAb, and showed a similar distribution of EGFP ${ }^{+}$cells (brown). Magnification $\times 200$. (i) Kidney sections from EGFP chimeric rats with WT BMC show $\mathrm{EGFP}^{+}$parenchymal structure with $\mathrm{EGFP}^{-}$nonparenchymal cells. All samples obtained 100 days after WBI and BMC infusion. Magnification $\times 400$.

With prolonged 18-h cold preservation, more prominent tubular epithelial cell injury was observed. As seen with warm I/R injury, a significant amount of tubular epithelial cell death, apoptotic debris, protein casts, tubular dilatation, and inflammatory infiltrates were seen (Figure 2c). At 30 days, there was persistent chronic interstitial inflammation and patchy tubular atrophy (Figure 2f).

\section{BM-Derived Tubular Epithelial Cells after Warm I/R Injury in Radiation Chimeras}

To examine the involvement of BM-derived cells in the repair process following I/R injury, the existence and frequency of tubular epithelial cells with BMC donor phenotype were examined in radiation chimeras after the induction of renal warm I/R injury. In WT recipients of $\mathrm{EGFP}^{+} \mathrm{BMC}$, three-color 
Table 1 Frequencies of tubules with BM-derived EGFP+/F-actin ${ }^{+}$cells after renal I/R injury

\begin{tabular}{|c|c|c|c|c|c|c|}
\hline \multirow[t]{2}{*}{ Model } & \multirow[t]{2}{*}{ Injury } & \multicolumn{2}{|c|}{ Serum creatinine level (mg/dl) } & \multicolumn{3}{|c|}{$\begin{array}{c}\text { Frequency of } E G F P^{+} / F \text {-actin }{ }^{+} \text {tubules }{ }^{\mathrm{a}}(\%) \\
\text { (expected frequency) }\end{array}$} \\
\hline & & $24 h$ & 30 days & $\begin{array}{l}\text { Pre I/R } \\
\text { injury }\end{array}$ & 7 days & 30 days \\
\hline Chimera $^{\mathrm{b}}$ & 60-min warm I/R & $3.72 \pm 1.11$ & $0.68 \pm 0.09$ & 0 & $0.50 \pm 0.23(0.12 \pm 0.06)$ & $0.17 \pm 0.06(0.04 \pm 0.02)$ \\
\hline WT to EGFP KTx & Minimum cold I/R & $1.10 \pm 0.01$ & $0.70 \pm 0.10$ & N/A & $0.18 \pm 0.13(0.05 \pm 0.03)$ & $0.08 \pm 0.07(0.02 \pm 0.02)$ \\
\hline WT to EGFP KTx & 18-h cold I/R & $4.33 \pm 0.31$ & $1.40 \pm 0.10$ & N/A & $0.65 \pm 0.49(0.16 \pm 0.20)$ & $0.17 \pm 0.19(0.04 \pm 0.05)$ \\
\hline
\end{tabular}

Mean \pm s.d. of 4-6 animals in each group.

${ }^{\mathrm{a}}$ The frequency of tubules with BM-derived $\mathrm{EGFP}^{+} / \mathrm{F}$-actin ${ }^{+}$cells was determined in 250-1100 tubules per animal in 2D random fields of the renal outer medulla. Expected frequency was calculated based on 3D analysis.

${ }^{\mathrm{b}}$ Radiation chimera was created by EGFP BMC infusion into WT rats after 9.5 Gy WBI, and I/R injury was induced 100 days later.
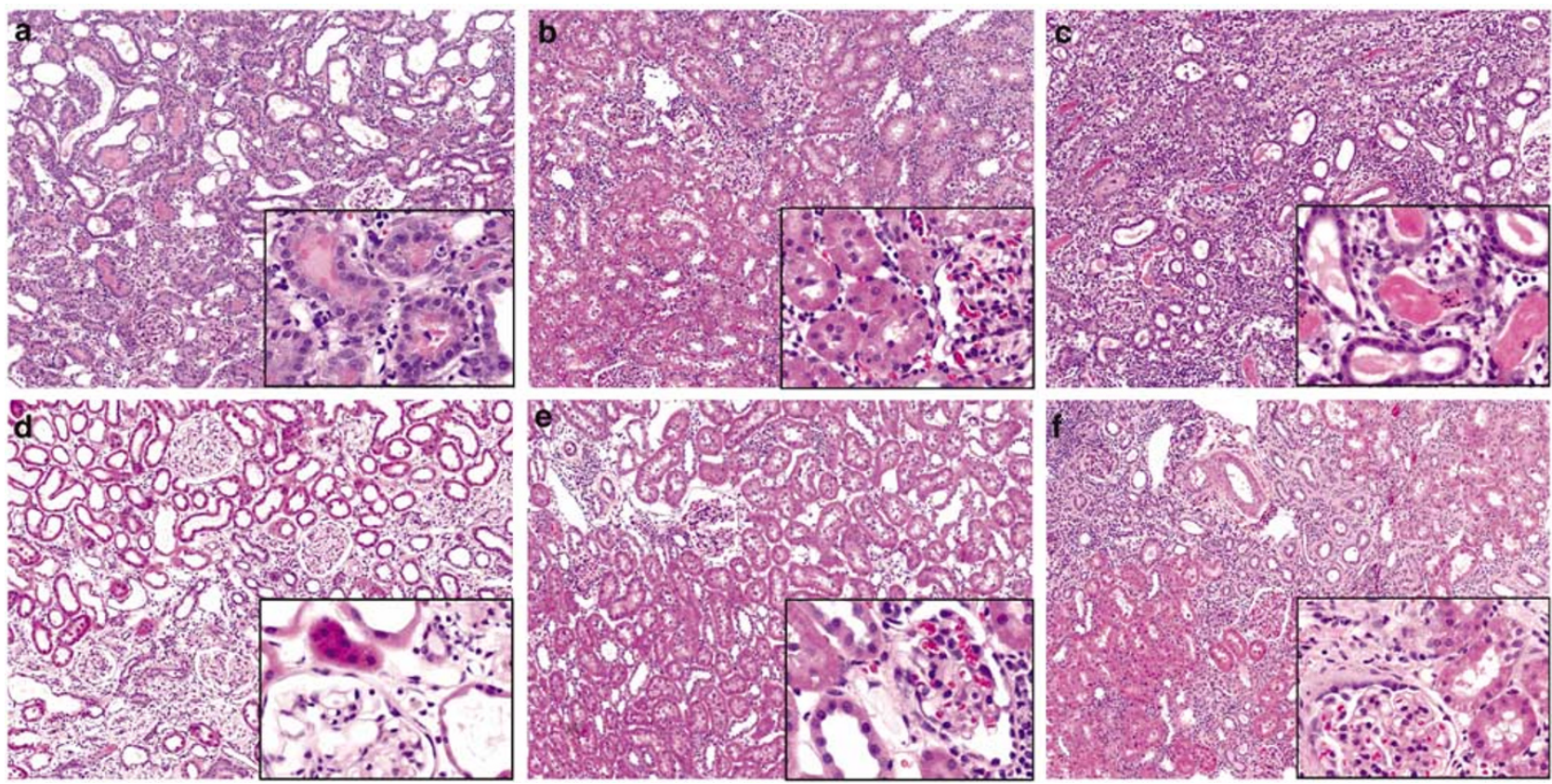

Figure 2 Routine histopathology of renal warm or cold I/R injury. H\&E sections of the kidney were obtained 7 days (a-c) and 30 days (d-f) after I/R injury. (a, d) Warm I/R injury, (b, e) KTx with minimal cold preservation, (c, f) KTx with $18 \mathrm{~h}$ preservation. At 7 days after I/R injury (a-c), tubular epithelial cell injury is seen as epithelial cell death/apoptosis, intraluminal necrotic cell debris, protein casts, and tubular dilatation. Mild to moderate interstitial infiltrates are also present. The histopathological severity of injury in general is 'cold I/R with 18-h preservation' > '60-min warm I/R' > 'cold I/R with minimal preservation', and correlates to serum creatinine levels (see Table 1). By 30 days (d-f), the majority of active injury subsides; however, variable tubular dilatation, atrophy, and inflammatory infiltrates persist in the former two models. Original magnification $\times 100$, insert $\times 400$.

immunofluorescence analysis with 2D fluorescent microscopy revealed the presence of few $\mathrm{EGFP}^{+} /$ F-actin ${ }^{+} /$Hoechst $^{+}$tubular epithelial cells at 7 days (Figure 3). Interestingly, these $\mathrm{EGFP}^{+}$tubular epithelial cells were identified as isolated singlecell integration or a cluster of maximum 2-3 cells. There was no tubule that was entirely organized with EGFP $^{+}$cells. EGFP $^{+}$tubular epithelial cells were also found at 30 days after I/R injury with a similar pattern; however, the frequency was less than that found after 7 days. Using 2D fluorescence microscopy, frequencies of renal tubules containing $\mathrm{EGFP}^{+} / \mathrm{F}$-actin ${ }^{+} / \mathrm{Hoechst}^{+}$epithelial cells were 0.50 and $0.17 \%$ at 7 and 30 days, respectively (Table 1).
Alternatively, in radiation chimeras of a reverse direction (EGFP recipients of WT BMC), detection of WT BM-derived renal tubular epithelial cells after warm I/R injury was relatively easier by identifying EGFP $^{-} / \mathrm{F}_{\text {-actin }}{ }^{+} /$Hoechst $^{+}$cells among overwhelming numbers of $\mathrm{EGFP}^{+} / \mathrm{F}$-actin ${ }^{+} / \mathrm{Hoechst}^{+}$tubules (Figure 4). Frequencies of tubules with BM-derived epithelial cells were $0.64 \pm 0.23 \%$ at 7 days, resulting in the similar incidence seen in WT chimeras of EGFP BMC. It is extremely difficult to prove that the EGFP transgene is expressed in every renal tubular epithelial cell in EGFP transgenic rats and that EGFP $^{-}$tubular epithelial cells in this experiment were derived from WT BMC. However, we have not seen tubular epithelial cells that do not express 

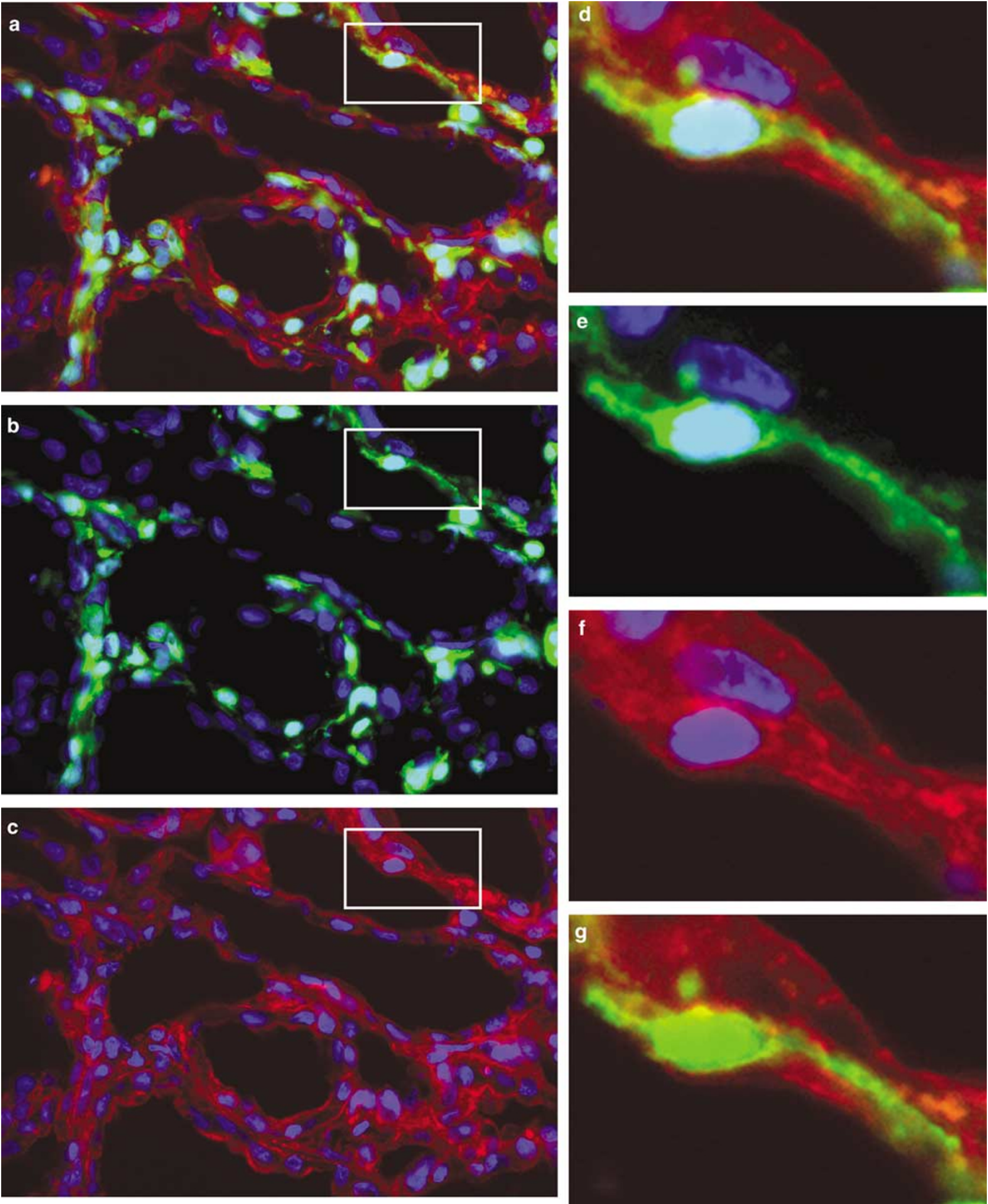

Figure 3 BM-derived EGFP ${ }^{+}$tubular epithelial cells after warm I/R injury. Renal warm I/R injury was induced in WT chimeras of EGFP BMC, and kidney sections were obtained at 7 days. (a) The cytoskeleton was stained with rhodamine phalloidin (F-actin, red) and the

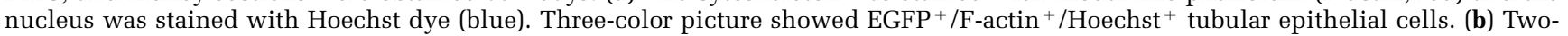
color image of (a) with nuclear stain and EGFP showed EGFP cytoplasmic expression. (c) Two-color image of (a) with F-actin and nuclear stain visualized tubular structures. (d-f) Magnification of BM-derived (EGFP ${ }^{+}$) tubular epithelial cells in (a). (d) Three-color image of BM-derived EGFP ${ }^{+} /$F-actin $^{+} /$Hoechst $^{+}$tubular epithelial cells. (e) Two-color image of (d), showing EGFP cytoplasmic expression with nuclear stain. (f) Two-color image of (d) with F-actin and nuclear stain. (g) Two-color image of (d), showing colocalization of EGFP and F-actin. Original magnification $\times 200(\mathbf{a}-\mathbf{c}), \times 800(\mathbf{d}-\mathbf{g})$. 

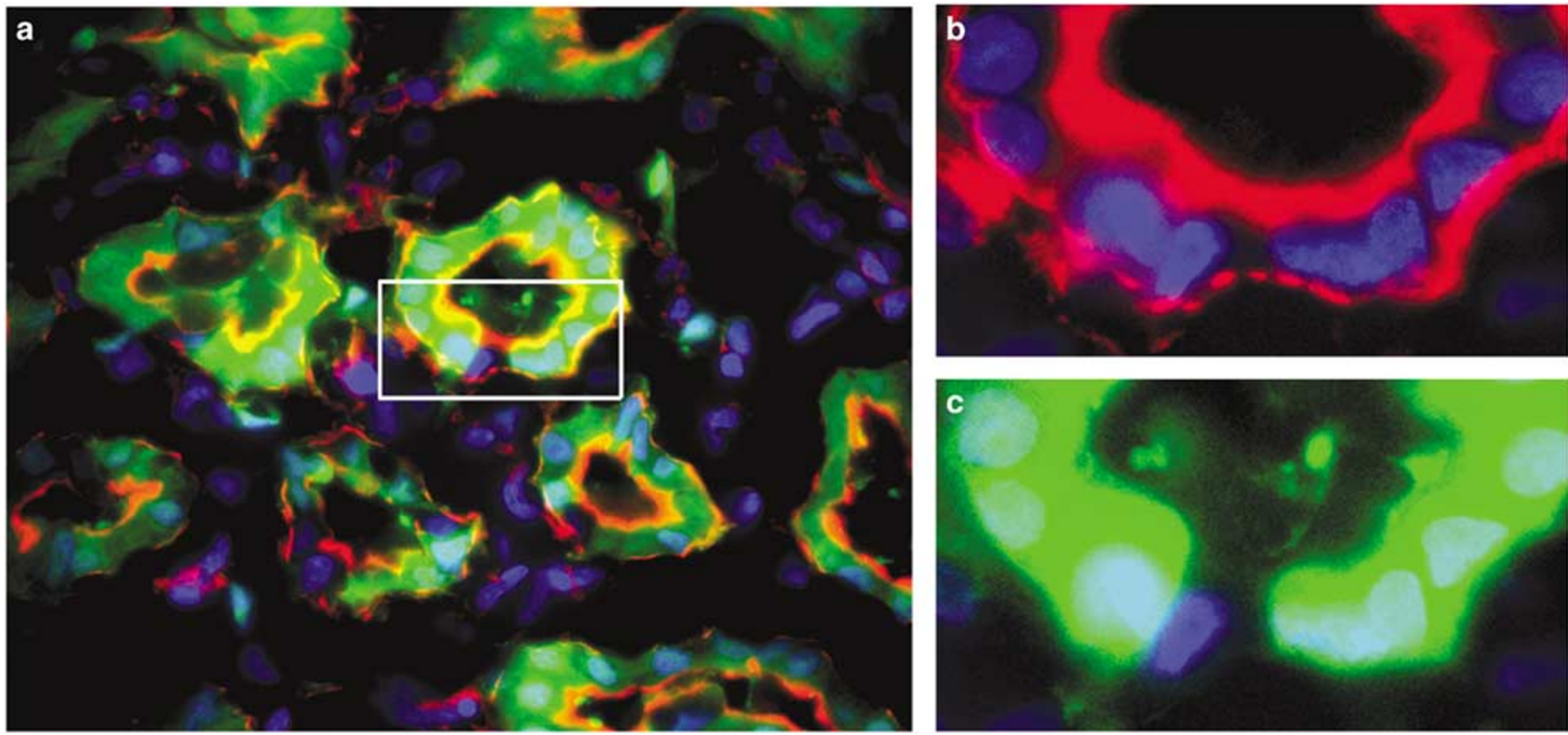

Figure 4 BM-derived EGFP- tubular epithelial cells after warm I/R injury. EGFP rats of WT BMC underwent renal warm I/R injury and kidney sections were obtained at 7 days. (a) Three-color image of EGFP, F-actin (rhodamine phalloidin, red), and nuclear stain (Hoechst dye, blue) showed EGFP ${ }^{-} / \mathrm{F}_{\text {-actin }}{ }^{+} /$Hoechst $^{+}$tubular epithelial cells. (b) Magnification of tubular structure in (a) with F-actin and nuclear stain. (c) EGFP ${ }^{-}$WT BM-derived tubular epithelial cells in two-color image of EGFP and nuclear stain. Original magnification $\times 200(\mathbf{a}), \times 600(\mathbf{b}, \mathbf{c})$

EGFP in naïve EGFP transgenic rats, while EGFP ${ }^{-}$ tubular epithelial cells were easily detected in experimental animals in this study. The frequency of $\mathrm{EGFP}^{-}$tubular epithelial cells was similar to that of $\mathrm{EGFP}^{+}$tubular epithelial cells seen in the reversed EGFP to WT model. Therefore, although it is not definitive, $\mathrm{EGFP}^{-}$tubular epithelial cells in WT to EGFP chimeras were most likely WT BMderived tubular epithelial cells.

\section{Host BM-Derived Tubular Epithelial Cells in Transplant-Induced Cold I/R Injury}

At 7 days after WT to EGFP rat KTx with minimum preservation, rare host $\mathrm{BM}$-derived $\mathrm{EGFP}^{+} /$ F-actin ${ }^{+} /$Hoechst $^{+}$tubular epithelial cells were identified as isolated cells in the tubules in the 2D images. The frequency of tubules containing $\mathrm{EGFP}^{+} / \mathrm{F}$-actin ${ }^{+} / \mathrm{Hoechst}^{+}$epithelial cells was $0.18 \pm 0.13 \%$. In contrast, when WT kidney grafts were preserved for $18 \mathrm{~h}$ and transplanted into EGFP rats, frequencies significantly increased at 7 days to $0.65 \pm 0.49 \%$, suggesting that the severity of injury may influence differentiation of BMC to renal epithelial cells. At 30 days, $\mathrm{EGFP}^{+}$/ F-actin ${ }^{+} /$Hoechst $^{+}$tubular epithelial cells markedly decreased compared to samples obtained at 7 days. One of four $(25 \%)$ rat recipients of KTx without cold preservation and three of six $(50 \%)$ recipients with 18-h UW cold preservation did not show any $\mathrm{EGFP}^{+}$tubular cells at 30 days after transplantation.

\section{Confocal 3D Imaging Using 50- $\mu \mathrm{m}$-Thick Sections}

To confirm the colocalization of EGFP, F-actin, and Hoechst dye on the tubular epithelium in $2 \mathrm{D}$ fluorescence microscope analysis, 3D analysis was conducted with a confocal scanning microscope using $50-\mu \mathrm{m}$ kidney sections. In radiation chimeras (WT recipients of EGFP BMC), suspected BMderived tubular epithelial cells $\left(\mathrm{EGFP}^{+} / \mathrm{F}^{-a} \mathrm{actin}^{+} /\right.$ DRAQ5 ${ }^{+}$) after warm I/R injury were targeted and analyzed with confocal microscopy by serially slicing at $0.3-\mu \mathrm{m}$ intervals. Sequential $2 \mathrm{D}$ images (typically 50-80 per case) were collected and processed to construct 3D projections. Established 3D images were viewed from several different angles. Results showed that 2D images frequently misrepresented the 3D distribution of the markers, and overlapping cells that were separately stained with different markers easily produced artifacts (false positive) in the 2D images. As seen in Figure $5 a$ and d, both 2D images demonstrated colocalization of EGFP and F-actin; however, 3D construction and careful assessment from different view angles revealed that true colocalization of EGFP and F-actin was only seen in Figure 5a-c. In contrast, the image in Figure $5 \mathrm{~d}$ was a misrepresentation of EGFP $^{+}$cells overlapping with $\mathrm{F}$-actin ${ }^{+}$epithelial cells, as seen from different angles in Figure 5e and $\mathrm{f}$. These different outcomes were more clearly seen in $3 \mathrm{D}$ viewing of Movie 1 and 2.

To further confirm the colocalization of markers on the single target cell, a 3D view was created with EGFP, F-actin, and nuclear DRAQ5 (Figure 6, Movie 

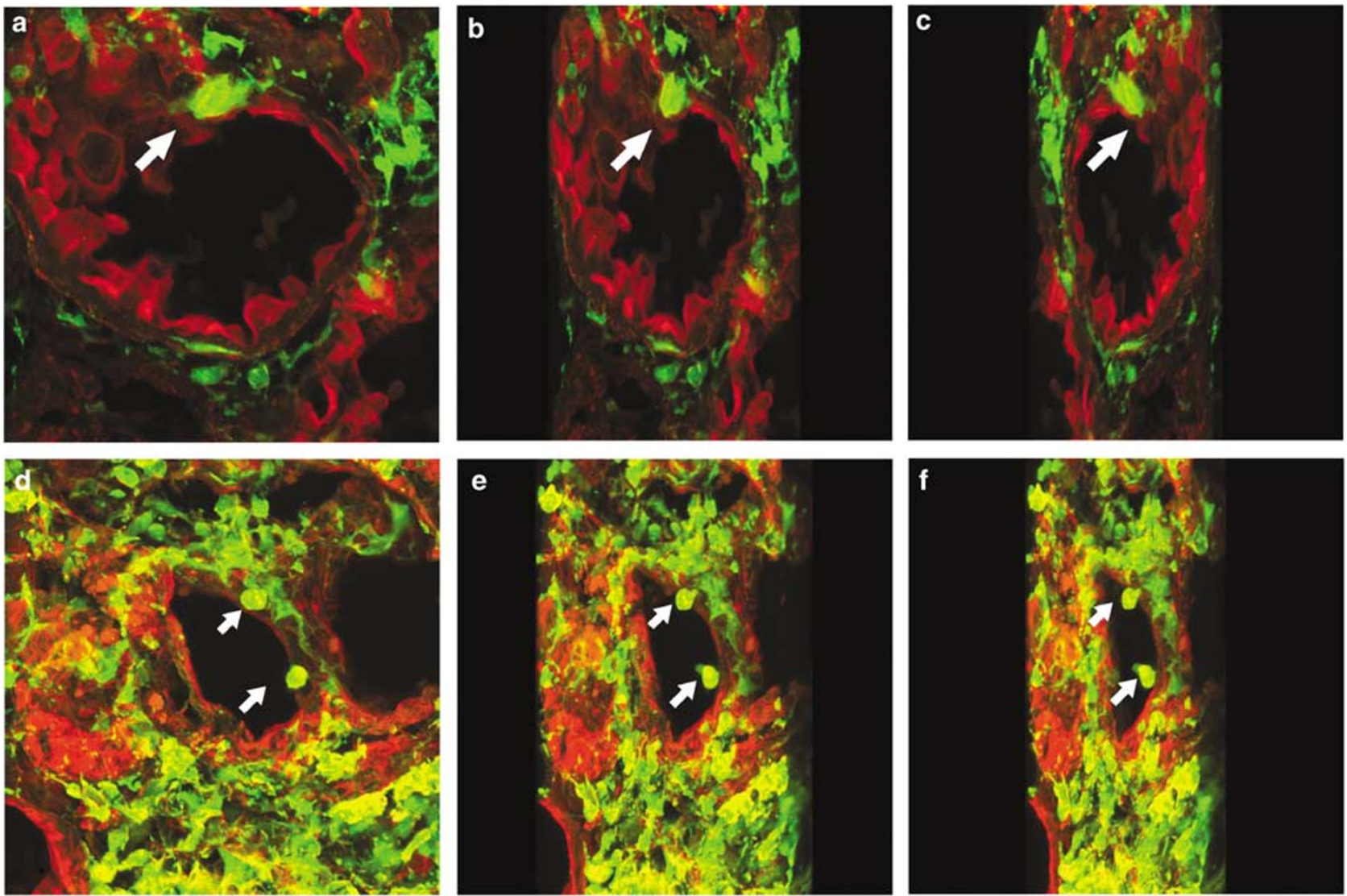

Figure 5 Several angles of pictures from 3D animation of 50- $\mu \mathrm{m}$ sections with rhodamine stain (see also Movies 1 and 2). Two 50- $\mu \mathrm{m}$ sections were stained with rhodamine phalloidin in order to visualize F-actin. (a-c) Different view angles showed BM-derived EGFP $+/$ F-actin ${ }^{+}$tubular epithelial cells (arrow). (e,f) Example of a false-positive case. Two EGFP ${ }^{+}$tubular cells seen in (d) were false (arrow). 3D construction and different view angles in (e) and (f) showed that $\mathrm{EGFP}^{+}$cells were inside of the tubular lumen. All samples were obtained 7 days after warm I/R injury in WT radiation chimeras of $\mathrm{EGFP}^{+} \mathrm{BMC}$

3). This 3D image with nuclear dye ascertained the generation of BM-derived tubular epithelial cells after I/R injury. Figure 6a-c (2D images from Movie 3) demonstrated that two tubular epithelial cells in this figure were regenerated from $\mathrm{BMC}\left(\mathrm{EGFP}^{+}\right.$/ F-actin ${ }^{+}$/DRAQ5 ${ }^{+}$cells, arrows), while the lower part of the same tubule did not show regeneration after warm I/R injury (arrowhead, Figure 6).

After extensive 3D reviewing of target cells selected in 2D images, we found that false-positive cases could be as frequent as $75 \%$, resulting in approximately $25 \%$ of true colocalization of EGFP, F-actin, and nuclear DRAQ5 stains in 3D analysis (Table 1).

\section{Discussion}

A large number of recent studies have shown that adult BMC can transdifferentiate into parenchymal cells and contribute to the repair of injured parenchyma. Rapidly growing numbers of clinical and experimental studies in a variety of diseases and models demonstrate the plasticity of BMC, and it becomes difficult to cite a complete list of literature. $^{12,13}$ In the kidney, BMC have been shown to generate mesangial cells, ${ }^{7,14,15}$ vascular endothelial cells, ${ }^{14,16}$ and tubular epithelial cells. The differentiation of BMC into renal proximal tubular cells has been shown principally in two models. First, in sexmismatched (female donor to male recipient) clinical KTx, male host phenotype tubular epithelial cells have been identified. Using fluorescent in situ hybridization and immunohistochemistry, Y-chromosome-positive cells expressing tubular cell markers were discovered in female kidney allografts at frequencies of $\sim 1 \%{ }^{17}$ and as high as $20 \% .{ }^{18}$ In these clinical studies, the analysis timing after KTx varied from a few days to a few years, and higher incidences of host phenotype renal tubule detection appeared to associate with renal injuries (eg ATN). Second, 'transdifferentiation' was experimentally demonstrated by BMC infusion into irradiated animals with renal warm ischemic injury. Using Rosa26 mice, $\beta$-galactosidase-tagged BM-derived hematopoietic stem cells (lineage ${ }^{-} /$Sca- $^{+} / \mathrm{c}^{-k t^{+}}$ $\mathrm{BMC}$ ) were shown to differentiate into renal tubular cells with extremely high frequencies $(\sim 80 \%){ }^{19,20}$ 

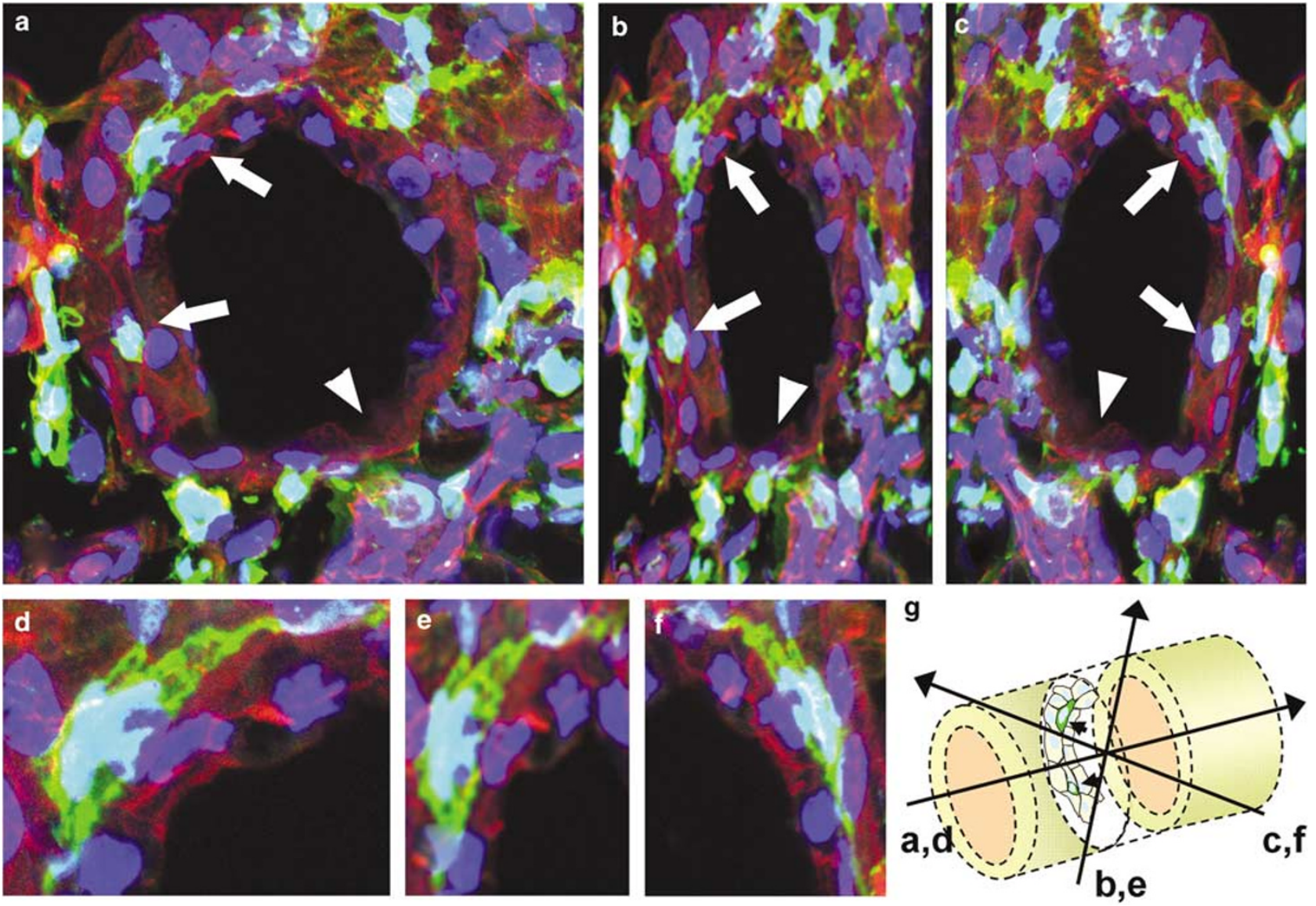

Figure 6 Several angles of pictures from 3D animation of 50- $\mu \mathrm{m}$ sections with F-actin/DRAQ5 nuclear stain (see also Movie 3). Kidney sections of 50- $\mu \mathrm{m}$ thickness were stained with rhodamine phalloidin in order to identify F-actin, and DRAQ5 nuclear dye. (a-c) Threecolor images from different view angles confirmed BM-derived tubular epithelial cells (EGFP $+/ \mathrm{F}_{\text {-actin }}+/ \mathrm{DRAQ5} 5^{+}$, arrows). Lower part of the same tubule (arrowhead) was not regenerated. (d-f) Magnification of BM-derived tubular epithelial cells in (a-c). (g) Scheme of two $\mathrm{EGFP}^{+} / \mathrm{F}^{-a c t i n}{ }^{+} / \mathrm{DRAQ}^{+}$cells in the tubule. Each arrow showed the view angle for (a-f).

In this study, using EGFP transgenic rats, we have examined differentiation of BMC into the renal tubules in both KTx and warm renal I/R injury models. Possible BM-derived tubular epithelial cells $\left(\mathrm{EGFP}^{+} / \mathrm{F}\right.$-actin $\left.{ }^{+} / \mathrm{DRAQ5}^{+}\right)$were selected in 2D three-color images and further analyzed with a confocal microscope by serially slicing the target cells at $0.3-\mu \mathrm{m}$ intervals and by constructing 3D projections. Using this stringent analytic method of 3D three-color immunohistochemistry, we confirm the emergence of BM-derived tubular epithelial cells colocalizing BM-derived EGFP, tubular epithelial cytoskeleton, and nuclear staining in kidneys from both models. However, the frequencies were unexpectedly low compared to the previous publications, and we encountered a high frequency of false-positive cases, in which positively identified cells in $2 \mathrm{D}$ analysis were indeed overlapping of two cells separately stained with different markers. The 3D analysis method used in this study revealed that BM-derived hematopoietic lineage cells (most likely inflammatory infiltrates) were closely located to renal tubules.
Thus, two types of cells with this proximity could be mistakenly characterized as BM-derived tubular epithelial cells when the 2D image was used as the sole detection method. After taking these false-positive cases into consideration, we estimated in this study that the frequency of renal tubules with BM-derived epithelial cells was approximately $0.1 \%$.

Section thickness and object size are important factors that can affect counting objects in histological sections. ${ }^{21-23}$ In general, quantitative analysis of thin sections is compromised by the fact that most cells are not wholly intact in thin sections, and the error becomes greater when the object size is larger relative to section thickness. In this study, we initially detected $\mathrm{EGFP}^{+} \mathrm{F}$-actin ${ }^{+}$ tubular epithelial cells in 6- $\mu \mathrm{m}$ cryostat sections, as this thickness was within the range of those used in other studies (commonly $4-8 \mu \mathrm{m}$ thickness sections). ${ }^{7,14-20}$ Therefore, methodologically, our 2D study using $6-\mu \mathrm{m}$ sections is comparable to other studies; however, the error rate might be different if other thicknesses are used. Subsequently, we 
conducted confocal microscopic analysis using $50-\mu \mathrm{m}$ sections. Since the average diameter of tubular epithelial cells is $15-35 \mu \mathrm{m}$, sections with $50-\mu \mathrm{m}$ thickness are required in confocal microscopy to visualize the whole structure of target tubular cells and to establish the relationship between the target and surrounding cells in the constructed 3D images. In fact, we were not able to obtain 3D images using $20-\mu \mathrm{m}$ or less thickness sections. Accordingly, the frequency of BM-derived tubular epithelial cells might be different between 6- and $50-\mu \mathrm{m}$ sections in this study. Partial sampling of targets in thin sections has been shown to result in an underestimation in quantification. ${ }^{23,24}$ Likewise, $\mathrm{EGFP}^{+} \mathrm{F}_{\text {-actin }}{ }^{+}$tubular epithelial cells in this study were relatively easily found in 50- than in $6-\mu \mathrm{m}$ sections; however, the difference was minor, and the frequency remained low in both thickness sections.

A number of studies describe transgene silencing, which reflects the diverse host genome defense responses via de novo DNA methylation or post-transcriptional sequence-specific RNA degradation. ${ }^{25}$ Silencing of EGFP transgene could be responsible for the low frequency of BM-derived tubular epithelial cells seen in this study. However, we found that the frequencies of BMC differentiation identified as $\mathrm{EGFP}^{+} / \mathrm{F}_{\text {-actin }}{ }^{+}$and $\mathrm{EGFP}^{-} / \mathrm{F}$-actin ${ }^{+}$tubular epithelial cells in EGFP to WT and WT to EGFP chimeras, respectively, were comparable, suggesting that EGFP transgene silencing would not be the case. Diverse frequencies of BMC-parenchymal cell transdifferentiation observed in clinical and experimental studies could be caused by the differences in species, severity of injury, replicative capability of progenitor cells or differentiated cells, and analytic methods. In addition, genetic modification might influence the outcome if experiments were performed in transgenic animals. Stringent and unequivocal detection systems, such as 3D structure analysis shown in this study, would be useful in exploring and understanding the involvement of BMC in tissue repair process. The value of $3 \mathrm{D}$ construction in immunopathological analysis was also recently reported by Spyridonidis et $a{ }^{24}$ demonstrating less frequent BMderived colon epithelium with $3 \mathrm{D}$ analysis in sex-mismatched human bone marrow transplant recipients.

\section{Acknowledgements}

We thank Mark Ross, Jason Devlin and Stuart Shand for excellent support in 3D imaging, Mike Tabacek and Lisa Chedwick for technical support, Masaru Okabe for providing EGFP rats, and Carla Forsythe for the preparation and organization of manuscript. This work was supported by NIH Grants DK54232, CA76541 and DK49615.

\section{References}

1 Pereira RF, Halford KW, O’Hara MD, et al. Cultured adherent cells from marrow can serve as long-lasting precursor cells for bone, cartilage, and lung in irradiated mice. Proc Natl Acad Sci USA 1995;92: 4857-4861.

2 Petersen BE, Bowen WC, Patrene KD, et al. Bone marrow as a potential source of hepatic oval cells. Science 1999;284:1168-1170.

3 McKay R. Stem cells in the central nervous system. Science 1997;276:66-71.

4 Krause DS, Theise ND, Collector MI, et al. Multi-organ, multi-lineage engraftment by a single bone marrowderived stem cell. Cell 2001;105:369-377.

5 Jiang Y, Jahagirdar BN, Reinhardt RL, et al. Pluripotency of mesenchymal stem cells derived from adult marrow. Nature 2002;418:41-49.

6 Okabe M, Ikawa M, Kominami K, et al. 'Green mice' as a source of ubiquitous green cells. FEBS Lett 1997;407: 313-319.

7 Ito T, Suzuki A, Imai E, et al. Bone marrow is a reservoir of repopulating mesangial cells during glomerular remodeling. J Am Soc Nephrol 2001; 12:2625-2635.

8 Murase N, Starzl TE, Tanabe M, et al. Variable chimerism, graft-versus-host disease, and tolerance after different kinds of cell and whole organ transplantation from Lewis to brown Norway rats. Transplantation 1995;60:158-171.

9 Adams AB, Durham MM, Kean L, et al. Costimulation blockade, busulfan, and bone marrow promote titratable macrochimerism, induce transplantation tolerance, and correct genetic hemoglobinopathies with minimal myelosuppression. J Immunol 2001;167: 1103-1111.

10 Kaizu T, Tamaki T, Tanaka M, et al. Preconditioning with tin-protoporphyrin IX attenuates ischemia/reperfusion injury in the rat kidney. Kidney Int 2003;63: 1393-1403.

11 Neto JS, Nakao A, Kimizuka K, et al. Protection of transplant-induced renal ischemia/reperfusion injury with carbon monoxide. Am J Physiol Renal Physiol 2004;3:3.

12 Herzog EL, Chai L, Krause DS. Plasticity of marrowderived stem cells. Blood 2003;102:3483-3493.

13 Korbling M, Estrov Z. Adult stem cells for tissue repair-a new therapeutic concept? N Engl J Med 2003;349:570-582.

14 Imasawa T, Utsunomiya Y, Kawamura T, et al. The potential of bone marrow-derived cells to differentiate to glomerular mesangial cells. J Am Soc Nephrol 2001;12:1401-1409.

15 Masuya M, Drake CJ, Fleming PA, et al. Hematopoietic origin of glomerular mesangial cells. Blood 2003;101: 2215-2218.

16 Rookmaaker MB, Smits AM, Tolboom H, et al. Bonemarrow-derived cells contribute to glomerular endothelial repair in experimental glomerulonephritis. Am J Pathol 2003;163:553-562.

17 Gupta S, Verfaillie C, Chmielewski D, et al. A role for extrarenal cells in the regeneration following acute renal failure. Kidney Int 2002;62:12851290.

18 Poulsom R, Forbes SJ, Hodivala-Dilke K, et al. Bone marrow contributes to renal parenchymal turnover and regeneration. J Pathol 2001;195:229-235. 
19 Lin F, Cordes K, Li L, et al. Hematopoietic stem cells contribute to the regeneration of renal tubules after renal ischemia-reperfusion injury in mice. J Am Soc Nephrol 2003;14:1188-1199.

20 Kale S, Karihaloo A, Clark PR, et al. Bone marrow stem cells contribute to repair of the ischemically injured renal tubule. J Clin Invest 2003;112:42-49.

21 Dhingra K, Sneige N, Pandita TK, et al. Quantitative analysis of chromosome in situ hybridization signal in paraffin-embedded tissue sections. Cytometry 1994;16: 100-112.

22 Guillery RW. On counting and counting errors. J Comp Neurol 2002;447:1-7.
23 Aubele M, Zitzelsberger H, Szucs S, et al. Comparative FISH analysis of numerical chromosome 7 abnormalities in 5-micron and 15-micron paraffin-embedded tissue sections from prostatic carcinoma. Histochem Cell Biol 1997;107:121-126.

24 Spyridonidis A, Schmitt-Graff A, Tomann T, et al. Epithelial tissue chimerism after human hematopoietic cell transplantation is a real phenomenon. Am J Pathol 2004;164:1147-1155.

25 Matzke MA, Mette MF, Matzke AJ. Transgene silencing by the host genome defense: implications for the evolution of epigenetic control mechanisms in plants and vertebrates. Plant Mol Biol 2000;43:401-415.

Supplementary Information accompanies the paper on Laboratory Investigation website (http://www. nature.com/labinvest) 